\title{
Références bibliographiques du dossier « les enseignants débutants »
}

Bernadette Plumelle

\section{OpenEdition}

12 Journals

Édition électronique

URL : https://journals.openedition.org/ries/5847

DOI : $10.4000 /$ ries. 5847

ISSN : 2261-4265

Éditeur

France Education international

Édition imprimée

Date de publication : 1 avril 2017

Pagination : 139-150

ISBN : 978-2-85420-614-2

ISSN : 1254-4590

Référence électronique

Bernadette Plumelle, "Références bibliographiques du dossier « les enseignants débutants » », Revue internationale d'éducation de Sèvres [En ligne], 74 | avril 2017, mis en ligne le 01 avril 2017, consulté le 11 mars 2022. URL : http://journals.openedition.org/ries/5847 ; DOI : https://doi.org/10.4000/ries. 5847 


\title{
Références bibliographiques du dossier " les enseignants débutants"
}

\author{
Bernadette Plumelle \\ Centre de ressources \\ et d'ingénierie documentaires, CIEP
}

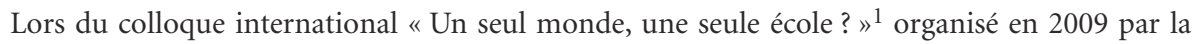
Revue internationale d'éducation de Sèvres, David Istance, du Centre pour la recherche et l'innovation dans l'enseignement (OCDE/CERI) rappelait l'un des scénarios développés par l'OCDE pour "l'école de demain » (2001) : une crise majeure liée au manque de professeurs donnant lieu à un exode et à un effondrement des systèmes d'enseignement. En 2017, la question des enseignants et de leur formation est à l'agenda de tous les pays. Les publications sur ce sujet se comptent par dizaine de milliers ; à titre d'exemple, le catalogue du centre de ressources et d'ingénieries documentaires du Centre international d'études pédagogiques (CIEP) en compte plusieurs milliers. Nous avons fait le choix de sélectionner des références récentes, majoritairement en langue française, en essayant d'équilibrer les études menées par les organisations internationales comme l'OCDE, l'Unesco, la Banque mondiale, etc., et les travaux de recherche. Ces références sont organisées thématiquement, sans distinction géographique, car les problématiques sont globalement partagées, qu'il s'agisse des pays à faible revenu ou de pays de l'OCDE : attractivité du métier d'enseignant, formation initiale, professionnalisation, décrochage enseignant, modalités innovantes dans la formation.

Les références proposées dans cette sélection complètent celles qui sont fournies par les auteurs des articles du dossier et ne sont pas reprises ici. Les résumés sont, pour l'essentiel, ceux des éditeurs ou des revues.

Bibliographie arrêtée le $1^{\text {er }}$ mars 2017.

\section{Une demande très forte de nouveaux enseignants au niveau mondial}

GAGNON Amélie, LEGAULT Elise, "Closing the primary teacher gap in subSaharan Africa: how many teachers are needed and how much would it cost? », Prospects, septembre 2015, $n^{\circ} 175$ - vol. XLV, $n^{\circ} 3$, p. 391-406 [en ligne]

Aujourd'hui, 58 millions d'enfants ne sont toujours pas scolarisés. L'un des préalables pour atteindre l'éducation pour tous est d'obtenir un nombre suffisant d'enseignants dans les classes. Depuis 2006, l'Institut de statistiques de l'Unesco fait des projections sur la demande mondiale d'enseignants. L'article présente les chiffres et la méthodologie utilisée ; il présente également des estimations des coûts associés au recrutement d'un nombre suffisant d'enseignants en Afrique subsaharienne pour atteindre l'éducation primaire universelle. [http://goo.g/ZnMMtv]

1. David ISTANCE, « Différentes perspectives sur les marchés de l'enseignement dans les travaux récents de l’OCDECERI ", Revue internationale de l'éducation - Sèvres, 2009, [en ligne], Colloque 2009 : Un seul monde, une seule école ? Les modèles scolaires à l'épreuve de la mondialisation [https://ries.revues.org/5693]. 

Bulletin d'information de l'ISU, octobre 2016, $n^{\circ} 39,16$. [en ligne]

À l'occasion de la Journée mondiale des enseignants, l'ISU fournit des estimations sur le nombre d'enseignants nécessaires pour réaliser l'Objectif de développement durable 4 sur une éducation inclusive et équitable de qualité. Au cours des 14 prochaines années, les pays devront recruter 68,8 millions enseignants pour permettre à chaque enfant d'accéder à l'enseignement : 24,4 millions pour le primaire et 44,4 millions pour le secondaire. L'ISU souligne les pénuries massives dans les cycles primaire et secondaire, principalement en Afrique subsaharienne, et établit des projections jusqu'en 2030. [http://goo.g//DbsYVU]

\section{RAYOU Patrick coordinateur, "Des enseignants pour demain : dossier », Éducation et sociétés, 2009, $n^{\circ} 23$, p. 5-168}

La profession enseignante connaît une crise profonde à l'échelle internationale : compressions budgétaires, influence des nouvelles technologies, marginalisation croissante d'une certaine partie de la jeunesse, crise du savoir, etc., avec pour conséquence des difficultés de recrutement et des situations de pénurie de professeurs dans certains pays. Ces facteurs ont une influence sur les missions et les habitudes professionnelles mais ont également ouvert des perspectives nouvelles aux enseignants. Les différentes contributions en font l'analyse selon trois axes : l'histoire du système éducatif et des politiques scolaires; le rôle des acteurs, les manières nouvelles d'exercer le métier d'enseignant et leur prise en compte par l'institution ; la dialectique entre l'individuel et le collectif dans les transformations actuelles.

UNESCO.IOS : Internal oversight service, A review of evaluative evidence on teacher policy, Unesco/Paris, mars 2016, 22 p. [en ligne]

Relever le double défi de l'offre et de la qualité des enseignants comme moyen de garantir la qualité de l'éducation constitue un programme ambitieux. Ce rapport est destiné à soutenir le discours sur la politique vis-à-vis des enseignants et pour s'assurer que les leçons apprises du passé se reflètent dans les futures politiques pour l'Agenda 2030. L'étude révèle que les organismes partenaires, tels que la Banque mondiale et l'Unicef, font la promotion de nombreuses stratégies et politiques pour aider les pays à relever ce défi. L'étude considère qu'il est urgent de diffuser les approches novatrices et d'institutionnaliser des politiques de recrutement et de maintien des enseignants qui soient sensibles au genre, la présence d'enseignantes pouvant considérablement améliorer la scolarisation des filles. [http://go0.g/05USVg]

\section{Attractivité (ou non) de la profession enseignante}

BERGER Jean-Louis, d'ASCOLI Yannick, "Les motivations à devenir enseignant : revue de la question chez les enseignants de première et deuxième carrière ", Revue française de pédagogie, $n^{\circ}$ 175, juin 2011, p. 113-146 [en ligne]

Les récents phénomènes de pénurie d'enseignants et de diminution de l'attrait pour ce métier amènent à s'interroger sur les motivations à devenir enseignant. Cette note synthétise les recherches qui ont abordé ces motivations, en se penchant non seulement sur les enseignants dont il s'agit de la première carrière, mais également sur ceux qui ont choisi le métier suite à une réorientation professionnelle. Les effets des diverses motivations sont ensuite analysés. [https://rfp.revues.org/3113] 
BRUNS Barbara, LUQUE Javier, Profesores excelentes : como mejorar el aprendizaje en América latina y el Caraibe, Banque mondiale, 2015, 360 p. [en ligne]

Les sept millions d'enseignants d'Amérique latine et des Caraïbes sont les acteurs essentiels de l'amélioration de la qualité de l'éducation. Cette étude analyse les enjeux économiques de la qualité des enseignants. Il expose les axes prioritaires de la réforme nécessaire pour former de bons enseignants : les politiques de recrutement; des programmes de formation des enseignants de meilleure qualité ; des incitations financières plus fortes pour motiver les enseignants tout au long de leur carrière. Un dernier chapitre analyse les politiques des dernières grandes réformes des enseignants au Chili, au Pérou, en Équateur et au Mexique. [https://goo.gl/osFG2e]

\section{CARLO Alain dir., MICHEL Alain coord., CHABANNE Jean-Charles, et al., Study} on policy measures to improve the attractiveness of the teaching profession in Europe - 2 volumes, Commission européenne/Bruxelles, 2013, 84 p. (vol. 1), 400 p. (vol. 2) [en ligne]

L'étude porte sur les enseignants de l'enseignement primaire et secondaire dans 32 pays européens. Après une description de l'état de la pénurie d'enseignants qualifiés, l'étude identifie les facteurs influençant l'attractivité du métier d'enseignant en distinguant ceux qui peuvent être plus faciles à modifier et ceux qui exigent des évolutions plus lentes à infléchir. Une typologie des politiques adoptées en Europe est ensuite présentée. [http://g0o.gl/90r3an], [http://goo.gl/z8scGW]

\section{CREHAN Lucy, Exploring the impact of career models on teacher motivation, UNESCO. IIPE/Paris, octobre 2016, 140 p.}

Si des enseignants qualifiés et motivés sont essentiels pour atteindre les objectifs de l'éducation pour tous à l'horizon de 2030, leur déroulement de carrière peut représenter un puissant levier pour améliorer leur motivation. Quelle est la relation entre la motivation des enseignants et le déroulement de leurs carrières? Quels modèles de déroulement de carrière existent dans le monde? Quels sont les plus efficaces? S'appuyant sur les expériences d'un large éventail de pays, l'ouvrage explore ces thématiques en identifiant différents modèles d'organisation de la carrière des enseignants et les défis liés à leur mise en œuvre. [https://goo.g/9Rq3e0]

MÜNICH Daniel, RIVKIN Steven, Analysis of incentices to raise the quality of instruction, Office des publications de l'Union européenne/Luxembourg, décembre 2015, 51 p. [en ligne]

Un récent rapport de la Commission européenne (2013) a montré le consensus qui règne autour du lien entre un personnel enseignant qualifié et motivé et la qualité de l'éducation dispensée. S'appuyant sur des travaux de l'OCDE, ce rapport analyse les implications de mécanismes d'incitation pécuniaires et non-pécuniaires sur la qualité de l'enseignement. Le rapport fait également état de la recherche concernant la formation initiale au métier d'enseignant dans les États membres de l'Union européenne et dans d'autres pays. Une synthèse en français de l'étude et de ses résultats est proposée p. 12-15. [http://goo.g//m100Zy]

\section{PÉRIER Pierre, Attractivité du métier d'enseignant : état des lieux et perspec-} tives, CNESCO/Paris, novembre 2016, 198 p. [en ligne]

Y a-t-il vraiment, en France, une pénurie de recrutement des enseignants ? Quel est le profil des étudiants souhaitant devenir enseignants? Leur image du métier, l'impact du salaire, du temps de travail, de la formation sur l'attractivité du métier? Que font les autres pays de l'OCDE pour valoriser le métier d'enseignant? Le rapport présente un état des lieux de cette question en France et dans les pays de l'OCDE. Il expose également les enjeux actuels et les politiques publiques d'attractivité menées dans quelques pays de l'OCDE. Le rapport s'achève sur une série de préconisations. [https://goo.g/EY21Jq] 


\title{
Formation initiale des enseignants
}

\author{
ALLEN Rebecca, BELFIELD Christopher, GREAVES Ellen, et al., The longer-term \\ costs and benefits of different initial teacher training routes, The institute for \\ fiscal studies/Londres, juillet 2016, 100 p. [en ligne]
}

En Angleterre, il existe diverses modalités de formation pour devenir enseignant. Chaque année, 35000 personnes peuvent choisir entre six parcours de formation qui varient, entre autres, par leur mode de financement. Actuellement, il y a un changement de politique de la formation initiale des enseignants. Ce rapport calcule le coût moyen par stagiaire des différentes formations pour l'administration centrale et pour les écoles. En outre, il fournit des données très récentes sur le maintien en poste des enseignants formés, selon le type de formation et en calcule la rentabilité à plus long terme. [https://goo.gl/yr8g2p]

ALTET Marguerite, DESJARDINS Julie, RICHARD Etienne, et al. (sous la direction de), Former des enseignants réflexifs : obstacles et résistances, De Boeck Université/Bruxelles, 2013, 286 p.

Alors que la formation et la professionnalisation du métier d'enseignant font l'objet de nombreuses publications, cet ouvrage collectif s'attache à mieux comprendre les rapports des étudiants à leur formation, notamment aux dispositifs qui visent à développer chez eux une posture réflexive. Cette prise de recul et de conscience ne va pas de soi et engendre le plus souvent scepticisme et résistance d'une partie des étudiants, des stagiaires et parfois des formateurs eux-mêmes. Les contributeurs partent de différentes hypothèses pour expliquer ces phénomènes ; ils font le constat de formes diverses de résistances et l'illustrent dans des contextes géographiques différents.

BOISSINOT Alain coordinateur, "Former des enseignants : dossier ", Revue internationale d'éducation de Sèvres, décembre 2010, $n^{\circ}$ 55, 172 p. [en ligne]

Ce numéro s'interroge sur la façon dont les pays définissent les grandes options qui inspirent leurs dispositifs de formation et dont ils organisent la formation initiale et le développement professionnel des maîtres. Aucun modèle stabilisé ne semble émerger, même si la formation des enseignants, partout considérée comme un enjeu majeur, tend souvent à être allongée et fait l'objet de réformes. La confrontation des analyses permet cependant d'identifier des problématiques récurrentes : la question des savoirs et de la professionnalisation; le rôle de l'État ; le choix des opérateurs ou la formation et le recrutement. [https://ries.revues.org/852]

\section{BOKDAM Johan, VAN DEN ENDE Inge, BROEK Simon, Enseigner aux ensei- gnants : situations et perspectives en matière de formation des enseignants $d u$ primaire en Europe, Parlement européen/Bruxelles, juillet 2014, 112 p. [en ligne] Dans quelle mesure les systèmes européens de formation des enseignants sont-ils actuellement adaptés à leur finalité ? Cette étude évalue la formation initiale en Europe, la prise en charge proposée en début de carrière et le perfectionnement professionnel continu. Elle analyse dans quelle mesure les stratégies nationales mises en place pour améliorer la qualité des enseignants sont liées aux différentes étapes de la carrière des enseignants et à une continuité dans la formation des enseignants. Les auteurs recommandent d'envisager la formation des enseignants comme un continuum. [https://goo.g/wtG4eR]}

JENSEN Ben, ROBERTS-HULL Katie, MAGEE Jacqueline, et al., Not so elementary: primary school teacher quality in top-performing systems, National Center on Education and the Economy /Washington DC, juillet 2016, 97 p. [en ligne]

La préparation des enseignants américains de l'enseignement primaire aux matières fondamentales est dans l'ensemble inadaptée. Ce rapport analyse la façon dont les systèmes éducatifs les plus performants soutiennent l'expertise disciplinaire de leurs enseignants. Les quatre 
contextes étudiés (Finlande, Shanghai, Hong-Kong et Japon) ont principalement recours à quatre politiques : a) sélectionner des candidats avec une forte expertise dans leur discipline ; b) exiger des enseignants qu'ils développent une expertise spécialisée dans une seule discipline ou dans quelques-unes; c) mettre l'accent sur une expertise approfondie des concepts fondamentaux de chaque discipline durant la formation initiale; $\mathrm{d}$ ) apporter un soutien spécifique aux disciplines au sein des établissements, grâce à des mentors et experts des disciplines ou du matériel didactique de qualité. [https://goo.gl/MhnpM5]

Commission européenne, Eurydice : réseau d'information sur l'éducation en Europe, "Initial teacher education and transition to the teaching profession ", dans The teaching profession in Europe: practices perceptions and policies, Office des publications de l'Union européenne/Luxembourg, juin 2015, p. 33-54, [en ligne]

Le rapport d'Eurydice analyse la relation entre les politiques qui règlementent la profession d'enseignant et les attitudes, les pratiques et les perceptions des enseignants. Dans ce chapitre, une partie est consacrée aux caractéristiques de la formation initiale des enseignants de l'enseignement secondaire. La deuxième partie porte sur la transition vers la profession enseignante : règlements centraux sur les programmes d'initiation pour les enseignants nouvellement qualifiés, leur participation et les principaux facteurs qui influent sur leur participation et les types d'activités concernés. Des renseignements sur le mentorat sont également fournis. Une synthèse du rapport en français [http://goo.gl/1wdulK] donne un aperçu des principales conclusions. [http://goo.g//YnOUJ]

FRAISSE Emmanuel, SELLIER Michèle (coordinateurs), "Missions et formation des enseignants de demain : dossier ", Administration et éducation, $n^{\circ} 144,2014$, p. $5-154$

Les questions des missions des enseignants et de la formation des maîtres, surtout si elles sont saisies de manière prospective, doivent être insérées dans un contexte marqué par deux phénomènes déterminants : la crise du métier d'enseignant, particulièrement sensible au sein de la société française, et la dimension mondialisée des interrogations qu'elle soulève. Ce dossier s'interroge sur les missions à assigner en priorité aux enseignants aujourd'hui. Par comparaison avec quelques pays étrangers, les contributeurs s'efforcent de faire ressortir les particularités françaises et de mettre en évidence les points de blocage.

LEBOUVIER Bruno, OUITRE Florian (coordinateurs), "Activité du débutant? quelle activité pour débuter ? : dossier », Recherches en éducation, novembre 2016, hors série $n^{\circ}$ 9, $155 \mathrm{p}$. [en ligne]

Ce numéro prolonge les travaux d'un séminaire de recherche organisé par l'École supérieure du professorat et de l'éducation (ESPE) de l'académie de Nantes. Les travaux présentés portent sur l'activité des enseignants débutants et sur les activités de formation qui leur sont proposées. Ils permettent également d'examiner la fonctionnalité d'outils susceptibles d'aider à l'ajustement des processus de formation des enseignants développés dans les différentes institutions (lieu de stage, institut de formation, premier établissement, etc.). [https://goo.gl/zmRUdU]

RAYOU Patrick, RIA Luc, "Former les nouveaux enseignants. Autour des statuts, de l'organisation et des savoirs professionnels ", Éducation et sociétés, 2009, $n^{\circ} 23$, p. $79-90$

Les situations d'enseignement ont été profondément modifiées par l'ouverture de l'école à des élèves peu familiers des exigences liées à des scolarités de plus longue durée. Elles nécessitent de la part des enseignants chargés de les y faire réussir de nouvelles compétences professionnelles qui se construisent dans l'alternance entre des mises en situation d'exercice et leur reprise conceptuellement étayée. Des enquêtes sur l'entrée dans le métier de jeunes enseignants montrent des évolutions à l'œuvre. Les praticiens débutants acceptent d'autant plus de se 
confronter aux savoirs de la formation et de la recherche que l'accompagnement dont ils bénéficient part de la réalité de leur travail et leur permet d'entrevoir collégialement des manières de faire à la fois partagées et spécifiques de chacun.

\section{Les nouveaux enseignants : l'entrée dans le métier}

BALLEUX André, PEREZ-ROUX Thérèse (coordinateurs), "Transitions professionnelles et recompositions identitaires dans les métiers de l'enseignement et de l'éducation ", Recherches en éducation, juin 2011, $n^{\circ} 11,179$ p. [en ligne]

Ce numéro s'intéresse aux parcours professionnels et plus particulièrement aux processus de transition vécus par les individus lorsqu'ils entrent dans les métiers de l'enseignement, de la formation ou de l'éducation. Au-delà des différences au niveau des systèmes de formation en France et au Canada, ce qui fait le point commun entre ces novices de l'enseignement ou d'une autre fonction en éducation, c'est leur formation antérieure, souvent disciplinaire qui ne les conduisait pas naturellement vers la nouvelle option professionnelle. Les contributions s'organisent autour de deux entrées éclairant des formes de mobilité spécifiques : mobilités conduisant de l'exercice d'un métier hors de l'espace scolaire au métier d'enseignant et mobilités internes au monde de l'éducation. [https://goo.gl/aRPNWV]

BUZNIC-BOURGEACQ Pablo, GERARD Laetitia (sous la direction de), L'apprentissage du métier d'enseignant : constructions implicites, espaces informels et interfaces de formation, Presses universitaires de Caen/Caen, 2015, 249 p.

«Enseigner est un métier qui s'apprend » : cette formule constitue l'objet d'étude ici questionné. Qu'est-ce que les enseignants apprennent pour faire leur métier? Comment, où et avec qui apprennent-ils ? De la maternelle à l'université, à l'école publique ou privée, face aux apprenants, parmi les collègues ou en formation, en France, en Suisse, en Belgique, en Tunisie ou au Canada, cet ouvrage collectif propose un regard croisé sur l'apprentissage du métier d'enseignant. Afin d'embrasser la diversité et l'étendue de cet apprentissage, trois orientations sont mises à l'étude : les constructions implicites, les espaces informels et les interfaces de formation.

DE STERCKE Joachim coordinatrice, "Insertion professionnelle des enseignants débutants en francophonie. Dossier ", Éducation et formation, juin 2013, $n^{\circ}$ e-299, p. 5-115, [en ligne]

L'insertion professionnelle des enseignants débutants est une problématique complexe et internationale. De nombreuses recherches ont été conduites autour de cette thématique depuis plus de 40 ans, et chaque année des programmes d'induction plus ou moins formels et structurés voient le jour pour soutenir l'entrée en carrière des nouveaux enseignants. Ce numéro invite le lecteur à la découverte de quelques-unes des plus récentes recherches et initiatives de terrain menées en lien avec l'insertion professionnelle des enseignants débutants. [http://goo.g/hZ3zvL]

FARGES Géraldine, "Le statut social des enseignants français au prisme du renouvellement générationnel ", Revue européenne des sciences sociales, 2011, $n^{\circ} 49-1$, p. 157-178 [en ligne]

Cet article analyse les transformations du statut social des enseignants en France, en s'interrogeant sur les conséquences des nombreux départs à la retraite, au début du XXI ${ }^{\mathrm{e}}$ siècle, et des recrutements consécutifs de "nouveaux enseignants ». La valeur sociale des professions de l'enseignement, le niveau d'études, la profession du conjoint et la profession du père sont principalement étudiés, à l'appui de données statistiques. Les cohortes les plus jeunes bénéficient d'un "prestige » social et intellectuel moindre que les précédentes, alors même qu'elles comptent davantage d'enfants d'enseignants, professions intermédiaires, cadres ou professions libérales en leur sein. [https://ress.revues.org/884] 
LUSSI BORER Valérie, RIA LUc (sous la direction de), Apprendre à enseigner, PUF/Paris, 2016, 250 p.

Aujourd'hui, tous les pays cherchent à optimiser l'apprentissage professionnel des futurs enseignants pour les préparer à un métier en profonde mutation. Le défi est d'innover en proposant de nouvelles orientations et méthodes : formation disciplinaire et transversale; en institution ; sur le terrain scolaire; en présentiel et à distance ; autoformation et co-formation; à partir de l'activité débutante et à partir de l'activité experte. Cet ouvrage a pour ambition de dresser un panorama des problématiques, des outils, et des espaces impliqués dans l'apprentissage du travail enseignant, s'appuyant sur un ensemble de travaux de recherche sur des expérimentations innovantes qui visent à faire évoluer les modalités de formation.

PEREZ-ROUX Thérèse, GAL-PETITFAUX Nathalie, DERNAT Sylvain, et al., "Accompagnement des transitions professionnelles et dispositifs réflexifs en formation initiale et continue ", Questions vives. Recherches en éducation, 2015 [en ligne]

Les contributions de ce numéro thématique abordent les transitions professionnelles à partir d'une perspective interactionniste. L'enjeu consiste aussi à interroger, à l'échelle internationale, les moyens mis en place pour accompagner les transitions, et plus particulièrement les dispositifs à visée réflexive étudiés par la recherche en éducation. Le numéro est structuré autour de quatre entrées : étudiants (dans différents pays, secteurs et systèmes de formation), enseignants (français, québécois, chiliens) et formateurs. [http://goo.g/HdM8gx]

\section{PÉRIER Pierre, Professeurs débutants : les épreuves de l'enseignement, PUF/} Paris, 2014, 188 p.

Le métier d'enseignant est-il en crise ou définitivement en voie de mutation ? Comment peut-on être enseignant? L'auteur, qui a mené plusieurs enquêtes auprès de professeurs débutants dans l'enseignement secondaire en France, observe les évolutions en profondeur de ce métier. La première partie s'intéresse à l'analyse des trajectoires des enseignants débutants depuis leurs origines familiales jusqu'au choix du métier. La deuxième partie a pour objet les rapports des enseignants avec leurs élèves dans la classe, la manière dont ils se construisent et se redéfinissent et le sentiment de "vulnérabilité professionnelle " qu'ils peuvent éprouver. La dernière partie s'attache à identifier les ressources institutionnelles et personnelles mobilisées par l'enseignant pour se forger son métier.

TREMBLAY Nicole, DUMOULIN Catherine, GAGNON Mathieu, et al., "Les défis de la formation initiale des enseignants du primaire en milieu à risque ", McGill Journal of education, 2014, vol. 49, $n^{\circ} 2$, p. 307-328, [en ligne]

Les enseignants débutant leur carrière dans les écoles accueillant une forte proportion d'élèves issus des milieux à risque (MR) seraient plus susceptibles d'abandonner la profession. La formation initiale qui leur est offerte ne leur permet probablement pas de s'adapter à ce contexte difficile. Grâce à des entrevues réalisées auprès de futurs enseignants en stage et de leurs enseignants associés, cette recherche a permis d'identifier trois défis de la formation initiale : acquérir une compréhension critique de leur cheminement culturel et des limites de ce dernier ; intégrer leurs connaissances des particularités des MR dans leurs pratiques; réguler celles-ci au contexte de pauvreté. [https://goo.g/9naMtm]

\section{VAN NIEUWENHOVEN Catherine, CIVIDINI Monica, Quand l'étudiant devient enseignant : préparer et soutenir l'insertion professionnelle, Presses Universi- taires de Louvain/Louvain, 2016, 276 p. \\ Les débuts dans l'enseignement, dans le contexte actuel toujours plus complexe et exigeant, s'avèrent difficiles. Tous ne disposent pas des mêmes ressources ni du même soutien pour vivre au mieux cette transition. L'ouvrage fait suite au symposium « La transition entre la formation initiale et l'insertion professionnelle des enseignants » (Louvain-la-Neuve, 2014), durant lequel}


ont été partagés des recherches et des dispositifs innovants d'insertion en Belgique et au Québec. Il est question de la préparation et de l'accompagnement des «transitants », ces enseignants novices en transition entre la formation et le terrain.

\section{Abandons de postes}

KARSENTI Thierry, COLLIN Simon, DUMOUCHEL Gabriel, " Le décrochage enseignant : état des connaissances ». International Review of Education, 2013, $n^{\circ}$ 59, vol. 5, p. 549-568 [en ligne]

Alors qu'un certain nombre de pays s'inquiètent du manque d'enseignants, la question se pose de savoir pourquoi ils sont si nombreux à quitter la profession. Cette revue de la littérature, fondée sur l'analyse de 69 articles scientifiques, vise à proposer des éléments de réponse à cette question, en identifiant les facteurs pouvant intervenir dans le décrochage enseignant. Les facteurs recensés sont répartis en trois catégories (facteurs liés à la tâche enseignante, à la personne enseignante ou à l'environnement social), puis examinés dans la conclusion.

[https://goo.gluFmtzA]

LOTHAIRE Sandrine, DUMAY Xavier, DUPRIEZ Vincent, « Pourquoi les enseignants quittent-ils leur école? Revue de la littérature scientifique relative au turnover des enseignants ", Revue française de pédagogie, 2012, n 181, p. 99-126 [en ligne]

Dans de nombreux pays, des études se sont penchées sur la mobilité professionnelle qui caractérise aujourd'hui le métier d'enseignant et, en particulier, les enseignants qui débutent dans la profession. Cette note de synthèse vise à appréhender les raisons pour lesquelles certains enseignants souhaitent quitter leur lieu de travail ou décident de le faire, que ce soit pour se réorienter professionnellement ou pour exercer leur métier dans un autre établissement. Elle centre l'analyse de la mobilité professionnelle enseignante sur les établissements, en essayant de comprendre pourquoi certains sont plus exposés que d'autres au turn-over des enseignants. Elle fait apparaître le rôle de la composition sociale, ethnique et académique des établissements ainsi que leurs caractéristiques organisationnelles. [http://rfp.revues.org/3931]

\section{Ministère de la Fédération Wallonie-Bruxelles/Bruxelles, Parcours professionnels} des enseignants du secondaire en début de carrière : synergies statistiques, Ministère de la Fédération Wallonie-Bruxelles/Bruxelles, 2015, 38 p. [en ligne]

Cette publication analyse l'évolution sur cinq ans de la cohorte des enseignants du secondaire de la Fédération Wallonie-Bruxelles entrés dans la profession en 2006. Elle indique un taux de départ important des enseignants dans les cinq premières années. Au terme de la première année de pratique professionnelle, $25 \%$ des enseignants quittent cette carrière. Ce chiffre monte à $39 \%$ en moyenne au terme des cinq premières années. Au fil de la publication, ce taux est nuancé par des analyses croisées avec des informations contextuelles. [http://goo.gl/wQ4xw0]

ROJO Sébastien, MINIER Pauline, "Les facteurs de stress reconnus comme sources de l'abandon de la profession enseignante au Québec ", Éducation et francophonie, vol. XLIII.2 - automne 2015, p. 219-240 [en ligne]

Cet article est consacré à la présentation des résultats d'une étude portant sur les divers facteurs de stress reconnus par de jeunes enseignants du secondaire pouvant générer l'abandon prématuré de la profession. La question de l'abandon de la profession par les jeunes enseignants est liée aux défis auxquels ils sont confrontés dès leur entrée en exercice. Ces défis les dépassent et entraînent du stress négatif qui produit de l'insatisfaction, un sentiment d'incompétence et d'inconfort psychologique. L'étude tente de mettre en lumière le point de vue d'enseignants du secondaire encore en fonction mais ayant pensé à abandonner, et celui d'autres enseignants ayant abandonné ou s'étant réorientés professionnellement. [https:/goo.gl/FOUuA3] 
RATOVONDRAHONA Pascale, NORMANDEAU Simon, Attrition et besoins en enseignants au primaire en Afrique subsaharienne, ODSEF : Observatoire démographique et statistique de l'espace francophone, Note de recherche de l'ODSEF, juin 2013, 27 p., [en ligne]

Avec un taux de croissance de plus de $25 \%$ entre 2000 et 2010, l'Afrique subsaharienne connait la plus forte augmentation de la population d'âge scolaire dans le monde. Le nombre d'enfants en âge de fréquenter l'école primaire a augmenté de 27 millions au cours de cette décennie, ce qui entraîne une demande accrue d'enseignants. Ces derniers n'ont pas toujours reçu la formation pédagogique minimale requise et sont très souvent confrontés à des classes pléthoriques. Cette étude porte sur l'importance de l'attrition des enseignants, c'est-à-dire sur le nombre d'enseignants quittant la profession. Elle s'intéresse aux facteurs et aux caractéristiques de l'attrition, à son impact sur l'apprentissage des élèves et sur le corps enseignant, au recrutement des enseignants. [https://goo.gl/ACqqwU]

\title{
Professionnalisation du métier d'enseignant
}

\author{
BARRERA-PEDEMONTE Fabian, High-quality teacher professional development \\ and classroom teaching practices: evidence from Talis 2013, OCDE/Paris, \\ octobre 2016, 34 p., [en ligne]
}

Ce document examine dans quelle mesure un développement professionnel (DP) de qualité peut aider les enseignants à trouver des stratégies pour améliorer l'apprentissage des élèves en classe. S'appuyant sur les données de l'enquête internationale de l'OCDE sur les enseignants, TALIS 2013, l'auteur compare les différents contenus et la manière de bénéficier de ce développement professionnel. Les résultats suggèrent que le DP axé sur le curriculum augmente la probabilité pour les enseignants d'utiliser une variété de méthodes d'apprentissage. En outre, les enseignants qui déclarent avoir participé à des activités de développement professionnel mettent en œuvre un niveau élevé de collaboration entre enseignants. [https://goo.gl/553bTm]

BOLD Tessa, FILMER Deon, MARTIN Gayle, et al., What do teachers know and do? Does It Matter? Evidence from Primary Schools in Africa, The World Bank Group/Washington D.C., janvier 2017, 37 p. (Policy Research Working Paper) [en ligne]

La scolarisation a augmenté de façon générale au cours des 25 dernières années dans les pays à faible revenu. Toutefois, une grande partie des enfants terminent leurs études primaires sans avoir acquis les compétences de base. Le rapport constate qu'une grande partie des enseignants ne maîtrisent pas les programmes qu'ils enseignent, que leurs connaissances pédagogiques sont faibles et que l'utilisation de bonnes pratiques d'enseignement est rare, alors même que ces connaissances et compétences ont des effets très positifs sur les résultats des élèves. Le rapport souligne la nécessité de cette prise en compte dans la conception des politiques éducatives.

\section{GUIBERT Pascal, PÉRIER Pierre (sous la direction de), La socialisation profession-} nelle des enseignants $\boldsymbol{d u}$ secondaire, Presses universitaires de Rennes, 2012, 164 p.

Les contributions rassemblées dans cet ouvrage analysent, à partir d'approches théoriques et de terrains, les étapes et parcours qui caractérisent l'accès au métier d'enseignant et son exercice dans l'enseignement secondaire en France. Elles visent ainsi à mieux comprendre les expériences et épreuves vécues lors des débuts, et leur place dans un processus de socialisation professionnelle de plus en plus ouvert et indéterminé. Sans doute cet enjeu prend-il un relief particulier à un moment où le recrutement des enseignants se tarit en France comme ailleurs, et que l'efficacité des politiques de formation des professeurs des premier et second degrés est interrogée. 
LEFRESNE Florence, RAKOCEVIC Robert, « Le métier d'enseignant en Angleterre aux Pays-Bas et en Suède : les voies sinueuses d'une professionnalisation ", Éducation et formations, décembre 2016, $n^{\circ}$ 92, p. 7-34, [en ligne]

Assiste-t-on à une professionnalisation du métier d'enseignant? L'Angleterre, les Pays-Bas et la Suède illustrent la dynamique dans laquelle semblent s'être engagés bon nombre de systèmes éducatifs en Europe. On constate notamment une responsabilisation accrue des établissements scolaires, entraînés dans une démarche de véritable management des ressources contribuant à estomper les frontières entre les secteurs public et privé. Plus récemment, le constat d'une pénurie d'enseignants qualifiés et d'une faible attractivité du métier d'enseignant, voire, dans le cas de la Suède, d'une baisse des acquis des élèves, attise les débats sur la qualité de l'éducation. [https://goo.gli41MJ7]

MORICONI Gabriela, BELANGER Julie, Supporting teachers and schools to promote positive student behaviour in England and Ontario (Canada): Lessons for Latin America, OCDE/Paris, avril 2015, $n^{\circ} 116,60$ p. [en ligne]

Le document présente les conclusions d'études de cas menées sur les systèmes d'éducation en Angleterre et dans la province canadienne de l'Ontario, dans le cadre d'un projet de recherche. Ces études de cas examinent la façon dont des mesures systémiques, principalement dans quatre grands domaines (formation initiale des enseignants, développement professionnel, collaboration professionnelle et participation des parties prenantes), ont été mises en œuvre afin d'améliorer le climat scolaire et d'aider les enseignants à faire face aux problèmes de comportement de leurs élèves. [https://goo.gl/LkGHal]

\section{PORTELANCE Liliane, MARTINEAU Stéphane, MUKAMURERA Joséphine (sous} la direction de), Développement et persévérance professionnels dans l'enseignement : oui mais comment ?, Presses de l'Université du Québec/Québec, 2014, 242 p.

La formation des enseignants est à l'agenda de nombreux systèmes éducatifs. Le travail et les responsabilités des enseignants se sont alourdis en raison de contextes éducatifs plus complexes, de mutations culturelles et sociales et ont rendu nécessaire la professionnalisation de la formation initiale des enseignants. Pour assurer la qualité des systèmes éducatifs, il est crucial que le développement professionnel soit un processus continu; or cette possibilité n'est pas offerte à tous les enseignants débutants, en raison de difficiles conditions d'embauche et de l'abandon de leur profession par beaucoup d'enseignants. Les auteurs de cet ouvrage proposent des réflexions afin de favoriser l'insertion professionnelle des enseignants débutants par l'entremise de l'exploration théorique, des expériences de formation liées aux stages, des savoirs développés par une analyse réflexive, des représentations quant à la collaboration professionnelle et interprofessionnelle ou de l'analyse de programmes de soutien à l'insertion professionnelle.

\section{OCDE : Organisation de coopération et de développement économiques, Supporting} teacher professionalism: insights from TALIS 2013, OCDE/Paris, février 2016, 221 p. [en ligne].

Basé sur l'Enquête internationale sur les enseignants, l'enseignement et l'apprentissage (TALIS), ce rapport de l'OCDE a pour objet le professionnalisme des enseignant(e)s dans le monde, basé sur des composantes clés : le socle de connaissances, l'autonomie dans la prise de décision et l'engagement dans des réseaux de pairs. L'auteur démontre qu'un niveau élevé de professionnalisme chez les enseignant(e)s leur est bénéfique et en détaille les modalités possibles : développer des programmes d'initiation et de tutorat; assister les enseignant(e)s dans la mise en place d'activités de recherches individuelles ou collaboratives en classe ; encourager les enseignant $(\mathrm{e}) \mathrm{s}$ à participer aux réseaux de pairs, destinés à l'échange d'informations. [http://goo.gl/5JDANl] 
SCHLEICHER Andreas, Teaching excellence through professional learning ad policy reform: lessons from around the world: international summit on the teaching profession, OCDE/Paris, 2016, 94 p. [en ligne]

Ce rapport a été préparé pour le $6^{\mathrm{e}}$ sommet international sur la profession enseignante (SIPE) (Berlin, 2016) autour du thème du développement et de la formation professionnelle des enseignant(e)s. L'auteur décrit les connaissances, les compétences et les qualités communes aux enseignants les plus efficaces. Il examine les politiques et les pratiques qui aident les enseignants à acquérir ces outils, y compris les programmes d'induction et des programmes de tutorat, les activités de perfectionnement professionnel continu, les évaluations des étudiants, et la collaboration entre collègues et partenaires de l'éducation. [http://goo.g//QB5gJq]

\section{De nouvelles modalités de formations}

BITEYE Babacar, BRANDT-POMARES Pascale, « La massification de la formation des professeurs contractuels sénégalais : formation à distance et professionnalisation ", Distances et médiations des savoirs, 2016, $n^{\circ} 14$. [en ligne]

L'article fait état d'une recherche sur la dimension professionnalisante du dispositif de formation à distance des professeurs recrutés massivement et sans formation professionnelle initiale au Sénégal. Les auteurs considèrent l'activité de formation à distance comme source de développement de compétences professionnelles des enseignants recrutés sans formation initiale et comme une voie possible de leur professionnalisation, dans un contexte de massification d'enseignants non formés. [https://dms.revues.org/1411\#authors]

FILÂTRE Daniel, Vers un nouveau modèle de formation tout au long de la vie: rapport sur la formation continue - Comité national de suivi de la réforme de la formation des enseignants et personnels d'éducation, Ministère de l'éducation nationale de l'enseignement supérieur et de la recherche/Paris, Académie de Versailles, novembre 2016, 32 p. [en ligne]

Le rapport du Comité de suivi de la réforme de la formation des enseignants en France, prenant en compte les rapports internationaux sur ce sujet, propose un ensemble de recommandations. Celles-ci visent à construire progressivement un nouveau modèle de formation tout au long de la vie des enseignants et personnels d'éducation et s'organisent en trois parties : un ensemble de principes préalables aux orientations proposées ; une approche nouvelle sur le continuum de formation; des propositions pour élaborer ce modèle. Le comité invite à considérer la formation des enseignants comme un continuum, depuis la licence jusqu'à la $3^{\mathrm{e}}$ année de titularisation. [https://goo.gl/M4E8F7]

\section{PERRENOUD P., ALTET M., LESSARD C. \& PAQUAY L., Conflits de savoirs en} formation des enseignants. Entre savoirs issus de la recherche et savoirs issus de l'expérience, De Boeck/Bruxelles, 274 p., bibliogr.

Les différents contributeurs abordent un problème central de la formation des enseignants : les rapports difficiles entre savoirs des praticiens et savoirs issus de la recherche. Leur alliance ne va pas de soi, car ces savoirs diffèrent par leurs origines, leur langage et leur degré d'abstraction, leur rapport à l'action et leurs critères de validité. Ils sont portés par des acteurs différents, les enseignants et les chercheurs. Les formateurs d'enseignants sont souvent des médiateurs entre ces deux mondes. Cet ouvrage analyse les obstacles qui rendent difficiles les métissages entre savoirs. Il propose aussi des dispositifs, dans le cadre des réflexions contemporaines sur l'alternance, l'articulation théorie-pratique, l'apprentissage par problèmes et le développement de compétences. 
RIA Luc (sous la direction de), 1. Former les enseignants au XXI siècle : établissement formateur et vidéoformation, De Boeck/Bruxelles, 2015, 275 p., bibliogr. L'ouvrage propose des regards novateurs et des voies d'exploration, permettant de penser autrement la contribution de l'établissement scolaire dans la formation des enseignants ainsi que les apports de la vidéoformation dans l'analyse des pratiques enseignantes. L'établissement d'exercice est abordé comme un véritable espace où les enseignants développent ou modifient leur professionnalité. La vidéo, comme ressource à l'analyse du travail enseignant, est de plus en plus utilisée dans la formation. L'auteur présente de nombreux dispositifs, sources de transformations professionnelles. 